\title{
The retardation effects of lamellar slip or/and chain slip on void initiation during uniaxial stretching of oriented iPP
}

\author{
Baobao Chang ${ }^{\text {a, }}$, Honghui Shi ${ }^{\mathrm{a}}$, Man $\mathrm{He}^{\mathrm{a}}$, Konrad Schneider ${ }^{\mathrm{b}}$, Gert Heinrich ${ }^{\mathrm{b}, \mathrm{c}}$, Chuntai Liu ${ }^{\mathrm{a}}$, \\ Changyu Shen ${ }^{a}$ \\ ${ }^{a}$ Key Laboratory of Materials Processing and Mold, Ministry of Education, National Engineering Research Center for Advanced Polymer Processing Technology, \\ Zhengzhou University, Zhengzhou, 450002, China \\ ${ }^{\mathrm{b}}$ Leibniz-Institut für Polymerforschung Dresden, Dresden, D-01069, Germany \\ ${ }^{\mathrm{c}}$ Institut für Textilmaschinen und Textile Hochleistungswerkstofftechnik, Technische Universität Dresden, Dresden, D-01069, Germany
}

\section{A R T I C L E I N F O}

\section{Keywords:}

Cavitation

Lamellae slip

Chain slip

Isotactic polypropylene

\begin{abstract}
A B S T R A C T
Cavitation is frequently encountered during the deformation of semi-crystalline polymers. Although abundant works have been performed to reveal the cavitation behavior in detail, the relationship between void formation and the plastic deformation of crystalline phase is still unclear. In this work, by in-situ synchrotron X-ray scattering, the void formation and the slip process in crystalline phase during uniaxial stretching of oriented isotactic polypropylene (iPP) were investigated. Results proved clearly that lamellae slip or/and chain slip presents a retardation effect on void formation. As the Deviation Angle (DA, defined as the angle between stretching direction and lamellae normal) was $0^{\circ}$, voids were initiated at a Hencky strain $\left(\varepsilon_{H}\right)$ of 0.04 and the longitude direction of voids was perpendicular to lamellae normal. Meanwhile, no slip process happened when voids were induced. As DA was $15^{\circ}$, lamellae slip took place once the stretching was started and voids were formed at $\varepsilon_{H}$ of 0.09 . When DA was further enlarged to $30^{\circ}$ and $45^{\circ}$, both lamellae slip and chain slip could be found as voids were induced. The $\varepsilon_{H}$ of voids formation were 0.29 and 0.31 , respectively. Interestingly, it is worth noting that as DA was increased from $0^{\circ}$ to $45^{\circ}$, although the orientation degree of voids was decreased, the longitude direction of voids stayed perpendicular to the normal of lamellae. When DA was enlarged further to $90^{\circ}$, voids were formed at $\varepsilon_{H}$ of 0.1 and the longitude direction of voids coincided with lamellae normal. Additionally, neither lamellae slip nor chain slip existed once voids appeared. Combining the value of micro-strain of lamellae stacks and crystal lattice, it is proposed that voids were formed either in the amorphous phase on the normal side of lamellae $\left(0^{\circ} \leq \mathrm{DA} \leq 45^{\circ}\right)$ or in the amorphous phase on the lateral side of lamellae $\left(\mathrm{DA}=90^{\circ}\right)$.
\end{abstract}

\section{Introduction}

"Cavitation is the sudden, unstable expansion of a void or bubble within a liquid or solid subjected to a negative hydrostatic stress" [1]. In semi-crystalline polymer materials, voids formation takes place frequently when external mechanical loads are applied [2-7]. The visible sign of void formation is the stress-whitening phenomenon. Since voids formation correlates intimately with yielding, failure, and brittle-ductile transition of polymer materials, tremendous investigations have been carried out to uncover the root cause governing voids formation [5,7-9]. In addition, it is worth noting that stretching induced void formation has been widely utilized as a technological method to prepare microporous film or microporous hollow fiber used in
Lithium battery or Extracorporeal Membrane Oxygenation [9-11].

Generally, voids can be induced only in tension, but not in compression or shearing deformation [5]. During uniaxial stretching, based on the macroscopic critical strain of void formation, two types of cavitation behavior have been reported $[7,12,13]$. In the case of voids formed around the yielding point, their longitude direction is perpendicularly aligned to the stretching direction (SD) and then transfers gradually to SD with increasing strain; In the case of voids which arise at large strains, their longitude direction remains unchanged in SD through the whole deformation process.

Voids formation is influenced greatly either by the interior microstructure (crystallinity, crystal form, lamellae thickness, spherulite size, molecular weight, etc.) or the employed external stretching parameters

\footnotetext{
* Corresponding author.

E-mail address: bbchang@zzu.edu.cn (B. Chang).
} 
(stretching rate, stretching temperature, stretching ratio) [8,9,14-20]. For instance, depending on the lamellar thickness and stretching temperature, three modes of cavitation were identified in polybutene- 1 (PB-1) [17]. For the quenched sample with the thinnest lamellae, no cavitation appeared during stretching; For the sample with thicker lamellae and stretched at lower temperature, voids were initiated around the yielding point and reoriented in the following stretching process; In the case of samples with thinner lamellae and stretched at higher temperatures voids were induced without reorientation. In the case of isotactic polypropylene (iPP), the molecular weight of polymers alters significantly the initiation of the cavitation process at large strain [13]. A higher molecular weight leads to a higher critical stress triggering the cavitation and to more intensive voids formation. But the intensity of cavitation initiated at small strains alleviated with increasing iPP molecular weight. Chang et al. [14] investigated the influence of crystal form on void formation in iPP. The results showed that due to the peculiar lamellae twisting of $\beta$-iPP during stretching, the void number was enlarged with increasing content of $\beta$-iPP. The intensified cavitation behavior in $\beta$-iPP compared with $\alpha$-iPP was also reported by other researchers [3,21]. Lu et al. [15] discussed the influence of spherulite size on void formation in PB-1. It was proved that the size of the cavities was bigger in the sample with larger spherulites than the one with smaller spherulites under the circumstance that crystallinity, long spacing and crystalline lamellar thickness were the same. Rozanski and Galeski revealed that once the stabilizers, additives, and low molecular weight fractions are extracted by critical $\mathrm{CO}_{2}$ or a mixture of non-solvents, more intense cavitation will appear in iPP [22]. Moreover, the penetration of chloroform and hexane into the interlamellar regions of iPP led to a decrease of the cavitation intensity [23]. In the later work by Pawlark, Krajenta, and Galeski [24], it was found that the reduction of entanglements in the amorphous phase will enhance the cavitation behavior by inducing numerous voids with sizes from nano-to micro-meters in iPP. More detailed description about void formation in semi-crystalline polymers can be found in the reviews of Galeski et al. [4] and Men et al. [6].

Despite the fact that abundant studies have been performed, the molecular mechanism of void formation in semi-crystalline polymers remains still open for debate. The obstacle impeding further understanding lies in the fact that in addition to void formation, hierarchical structural evolution in the crystalline phase appears simultaneously during deformation [25-29]. For instance, Young et al. [25] defined three principle deformation modes (chain slip, lamella slip, and fibrillary slip) during stretching of oriented polyethylene (PE). The deformation system favored shearing parallel or perpendicular to the chain direction, and the elastic deformation is caused mainly by fully recoverable lamella shear. Otherwise, the permanent plastic deformation is a result of chain slip in the chain direction. Burnay et al. [26] examined the deformation of $\mathrm{PE}$ as a function of the orientation of the tensile axis with respect to the chain direction. Their results showed that slip processes parallel to the chain direction are the dominant modes over most of the orientation range. Moreover, Peterlin divided the crystal slip into either fine slip or coarse slip [27]. Fine slip gives rise to a change of the angle between chain axis and lamellae normal, whereas for coarse slip this angle remains unchanged. Employing the step-cycle run methodology, Strobl et al. [30-32] found four characteristic points during semi-crystalline deformation based on their recovery properties. These points are associated with (1) the onset of isolated slip processes, (2) a change into a collective activity of the slips, (3) the beginning of crystallite fragmentation, and (4) chain disentanglement resulting in a finite truly irreversible deformation.

The relationship between void formation and the hierarchical deformation process in crystalline phase was firstly addressed by Pawlak and Galeski [5] and then followed by Lame et al. [8]. Pawlak and Galeski [5] proposed that in tensile deformation exists a competition between cavitation and plastic deformation of the crystalline phase. Considering the stress concentration between lamellae, the absolute value of the negative pressure required for cavitation is $-p_{\text {cav }}<\sigma / 3$, and the necessary condition for chain slip is $2 \tau_{0} /(1-K)<\sigma$, where $p_{\text {cav }}$ is the negative pressure required for cavitation, $\sigma$ is the stress, $\tau_{0}$ is the plastic resistance of a crystal in simple shear and $K$ is the stress sensitivity factor. Then cavitation happens in polymers with crystal of higher plastic resistance, while crystal plastic deformation occurs in polymers with crystal of lower plastic resistance. Lame et al. [8] further proved that the stresses necessary to initiate cavitation and crystallite shearing relied respectively on stress transmitters (tie molecules, interphase, etc.) and crystallite thickness. Depending on the molecular topology and the microstructure, either cavitation or crystallite shearing can be the cause or the consequence of the other one.

Due to the discussion in the introduction, it can be concluded that the relationship between void formation and crystalline phase deformation is not fully understood yet. The role of the lamellae slip and/or the chain slip on void formation needs to be clarified. To get a deeper insight into the relationship between void formation and slip process in the crystalline phase, in this study the void formation in pre-oriented iPP during uniaxial stretching is monitored by in-situ synchrotron small angle X-ray scattering (SAXS). In addition, the slip process is recorded by in-situ synchrotron wide angle X-ray scattering (WAXS). By changing the angle between the stretching direction (SD) and the normal of lamellae (defined as the Deviation Angle, DA), distinct different cavitation behavior and crystalline phase slip processes are observed, and the relationship between them is here presented and discussed.

\section{Experimental section}

\subsection{Materials and sample preparation}

iPP used in this study is a homo polymer from Borealis (Linz, Austria) with a brand of HD120MO. The melt flow index is $8 \mathrm{~g} / 10$ min under ASTM D1238 $\left(230{ }^{\circ} \mathrm{C}\right.$ and $\left.2.16 \mathrm{~kg}\right)$. The weight-average and numberaverage molecular weights are $365 \mathrm{~kg} / \mathrm{mol}$ and $67.6 \mathrm{~kg} / \mathrm{mol}$, respectively. To control DA, the following two-step strategy was employed: in the first step, plates with a thickness of $1 \mathrm{~mm}$ were injection molded. The barrel temperature and mold temperature were $210{ }^{\circ} \mathrm{C}$ and $50{ }^{\circ} \mathrm{C}$, respectively; in the second step, a waist-shape specimen was cut from the injection molded plate by Computerized Numerical Control (CNC). The angle between the longitudinal axis of the specimen and the machine direction of the injection molded plate were $0^{\circ}, 15^{\circ}, 30^{\circ}, 45^{\circ}$, and $90^{\circ}$. For simplification, the specimens were labeled as PP0, PP15, PP30, PP45, and PP90 correspondingly.

\subsection{Uniaxial stretching and in-situ synchrotron $X$-ray scattering experiments}

The stretching of the waist-shape specimen was performed on a custom-made miniature tensile machine. The stretching speed was 0.02 $\mathrm{mm} / \mathrm{s}$, and a stretching temperature of $75{ }^{\circ} \mathrm{C}$ was adopted in coincide with our previous work $[12,14]$. Since that the specimen was deformed un-homogeneously in the stretching direction especially when necking happens (Fig. S1), the local strain $\left(\varepsilon_{H}\right)$ at the position where X-ray past was assessed by the change of the specimen width by

$\varepsilon_{H}=2 \ln \frac{b_{0}}{b}$

where $b_{0}$ and $b$ are the initial width and the width during stretching.

In-situ synchrotron X-ray scattering measurements were carried out at the MiNaXs Beamline at Deutsches Elektronen Synchrotron (Hamburg, Germany). The X-ray wavelength was $0.092475 \mathrm{~nm}$ and the exposure time was $0.1 \mathrm{~s}$. For two-dimensional (2D) small angle X-ray scattering (SAXS) and wide-angle X-ray scattering (WAXS), a Pilatus $1 \mathrm{M}$ detector $(981 \times 1043$ pixels $)$ and a Pilatus $300 \mathrm{~K}$ detector $(487 \times 619$ pixels) were used to record the pattern, respectively. The distances 
between the sample and the detector were set at $5706 \mathrm{~mm}$ and $210 \mathrm{~mm}$ correspondingly. The patterns were pre-processed by masking and background correction.

\subsection{Atomic force microscopy measurement}

The morphology of the specimen at the final stage of stretching was observed by Atomic Force Microscope (AFM) (Bruker MultiMode 8). A tapping mode was chosen. Before the measurement, the specimen was cut by Cryo-ultra-microtome (Leica EM FC7). The cutting temperature was set at $-80{ }^{\circ} \mathrm{C}$.

\section{Results and discussion}

The stress $(\sigma)$-strain $\left(\varepsilon_{H}\right)$ curves of the specimens are presented in Fig. 1a. Fig. $1 \mathrm{~b}$ is the enlargement of the square region in Fig. 1a. The vivid scene about the geometry evolution during deformation recorded by an optical CCD camera could be found in Fig. S1 in Supporting Information. Obviously, with the increase of $\varepsilon_{H}$, each curve can be divided into four regions: (i) a linear elastic deformation region $\left(0<\varepsilon_{H}<\varepsilon_{H l}\right)$, in this region $\sigma$ increased linearly with $\varepsilon_{H}$; (ii) a transition region $\left(\varepsilon_{H l}<\right.$ $\varepsilon_{H}<\varepsilon_{H s}$ ) located between the linear region and the strain softening region where the slope of $\sigma-\varepsilon_{H}$ curve decreased gradually and, finally, reached a plateau value; (iii) a strain softening region $\left(\varepsilon_{H s}<\varepsilon_{H}<\varepsilon_{H h}\right)$ where the slope of the $\sigma-\varepsilon_{H}$ curve remained constant; and (iv) a strain hardening region $\left(\varepsilon_{H}>\varepsilon_{H h}\right)$ where the slope of the $\sigma-\varepsilon_{H}$ curve increased again. The statistical method of $\varepsilon_{H l}$ and $\varepsilon_{H s}$ is shown in Fig. S2 in the Supporting Information. With increasing DA, $\varepsilon_{H l}$ stayed constant as $0.05-0.06$, but $\varepsilon_{H s}$ is changed obviously. $\varepsilon_{H s}$ is $0.11,0.14,0.15,0.16$, and 0.14 for PP0, PP15, PP30, PP45, and PP90.

\subsection{Void formation and its corresponding critical strain}

Representative 2D-SAXS patterns during stretching are presented in Fig. 2 whereby SD is horizontal. The grid on the pattern is caused by the beam stop holder and the gap between diode sensors. Before stretching, the pattern exhibited two-spots "water-drop" scattering as a result of ordered packing of lamellae stacks. For PP0, the spots were aligned on the equator, suggesting that the normal of lamellae was parallel to SD. As $\varepsilon_{H}$ was increased to 0.05 , a streak scattering arose in the center region of the pattern, indicating the formation of voids. Normally, an elliptic geometry was adopted for voids in deformed semi-crystalline polymers [33]. A horizontal alignment of the streak meant that the longitude axis of the void was in the vertical direction. When $\varepsilon_{H}$ was further enlarged to 0.20 , due to the continuous nucleation and growth of voids, the streak expanded in both horizontal and vertical direction, and then covers the lamellae scattering. In addition, a very weak vertical streak showed up, as a sign for the formation of a new group of voids. With the further increase of $\varepsilon_{H}$, the scattering increased mainly in the vertical direction. Meanwhile, the scattering in the horizontal direction shrinks moderately. The transformation of the scattering direction implied the orientation change of voids from perpendicular to parallel to the drawing direction, which was also evidenced in other works $[12,17,24$, 34].

For PP15, the spots scattering was inclined $c a .15^{\circ}$ to the horizontal direction. The streak scattering as a sign of voids formation appeared at a $\varepsilon_{H}$ of 0.1 , which was larger than that of PP0. What's more important, the streak was oriented in the same direction with the spots, suggesting that the long axis of voids was parallel to the normal of lamellae. With increasing $\varepsilon_{H}$, the intensity of the streak was enhanced. As $\varepsilon_{H}$ was larger than 0.3 , a new streak scattering could be found due to voids coalescence. Afterwards, the total scattering intensity of voids weakened moderately as the stretching continued.

For PP30, the lamellae scattering was inclined $c a .30^{\circ}$ to the horizontal direction. As $\varepsilon_{H}$ was larger than 0.3 , a visible rhombus-shape scattering shows up in the center of the pattern. And the direction of the rhombus-shape scattering was in line with the spots, but the orientation was quite weak. As $\varepsilon_{H}$ was further enlarged, the intensity of the rhombus-shape scattering was enhanced, and the intensity of the lamellae scattering was decreased due to stretching induced melting in crystalline phase [35]. At the end of stretching, the voids scattering evolved into a vertical streak.

For PP45, the lamellae scattering was located on the diagonal. The rhombus-shape void scattering appeared at a $\varepsilon_{H}$ of 0.4 , and the orientation of the rhombus was even weaker compared to PP30. As $\varepsilon_{H}$ was increased, the intensity of the void scattering was enhanced. And the geometry of the scattering changed into a vertically aligned streak at the end of stretching. For PP90, the lamellae scattering was positioned on the meridian. For $\varepsilon_{H}$ of 0.15 , the void scattering appeared in the form of a "butterfly-shape". The "wings" of this "butterfly" was positioned horizontally. The geometric transition of the void scattering from streak to butterfly indicated a decreased void orientation [33]. With continuous stretching, the void scattering grew gradually into an isotropic circle $\left(\varepsilon_{H}=0.3\right)$, and then further into a vertically aligned streak.

The height diagram of the specimen at the final stage of stretching is provided in Fig. 3, SD is horizontal. The image covers a range of $10 \times 10$ $\mu \mathrm{m}^{2}$. It is obvious that the size and orientation degree of voids are distinct different as DA is changed. In PP0, a laminated structure can be found. The dark region in the laminated structure is void. Voids are oriented perfectly along SD. Statistical analysis of the void size can be found in Fig. S3 in the Supporting Information. The average length and width of voids are $1250 \mathrm{~nm}$ and $130 \mathrm{~nm}$. The number of voids in the observed area is $c a$. 103. As DA is increased from 0 to $45^{\circ}$, the length and number of voids are reduced greatly. In addition, the orientation of voids becomes less perfect. In PP90, similar with PP0, voids are highly oriented in SD. But the length and width is much smaller than that in PP0, which are $570 \mathrm{~nm}$ and $60 \mathrm{~nm}$. The void number is much larger than that in PP0, which is ca. 280.

To get the critical strain of void formation $\left(\varepsilon_{H v}\right)$, the scattering invariant $(Q)$ is calculated according to the following equation
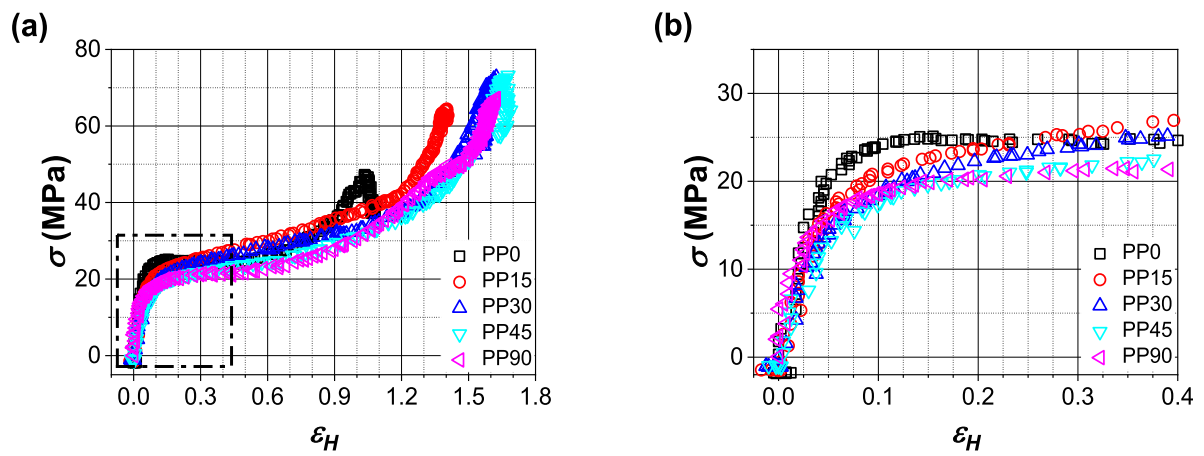

Fig. 1. (a) The stress $(\sigma)$-strain $\left(\varepsilon_{H}\right)$ curves of the sample with different lamellae orientation angle with respect to SD; (b) is the enlargement of the square region in (a). 

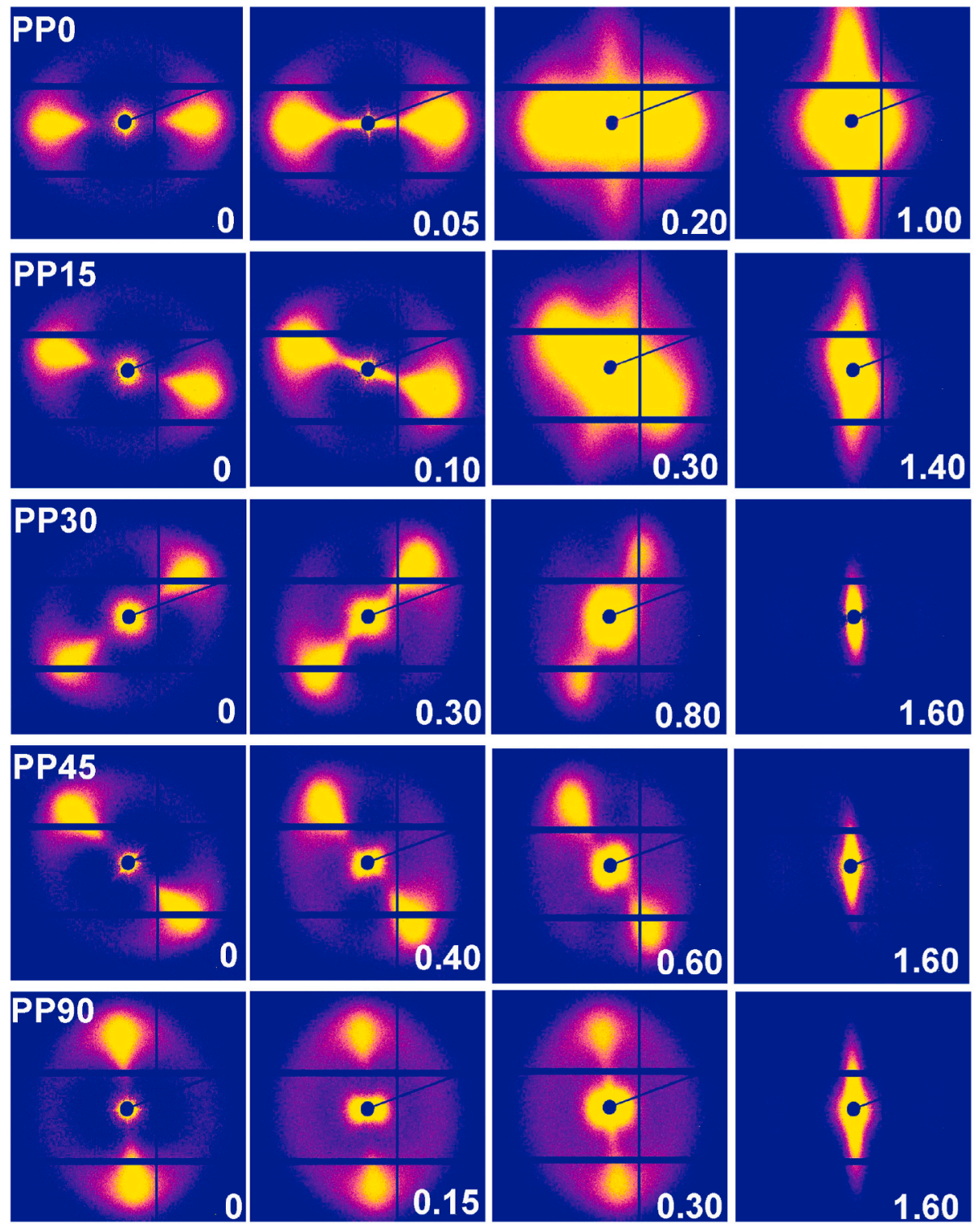

Fig. 2. The representative two-dimensional (2D) in-situ SAXS patterns during stretching. The numbers in the lower right side are $\varepsilon_{H}$. SD is horizontal.

$Q=\frac{1}{2} \int_{s_{\text {lower }}}^{S_{\text {upper }}} \int_{0}^{\pi} \cos (\phi) I(s) s^{2} d \phi d s$

with $\phi$ is the azimuthal angle and $s$ is the scattering vector. $s_{\text {lower }}$ and $s_{\text {upper }}$ are the lower and upper limitation of the scattering vector. During the SAXS measurement, the beam stop was placed in front of the detector, the scattering intensity in the center region of the pattern was blanked. So $s_{\min }$ was set at $0.0075 \mathrm{~nm}^{-1}$. In addition, to exclude the influence of lamellae scattering, $s_{\max }$ was chosen as $0.0160 \mathrm{~nm}^{-1}$. Assuming that the density difference $(\Delta \rho)$ between void and the matrix is constant, $Q$ is then a function of the volume fraction of voids $(V)$, i.e.

$Q=K * 2 \pi^{2} V(1-V) \Delta \rho^{2}$

where $K$ is a constant. Since $V$ is the only variable on the right side of the equation, changes of $Q$ could be used as an indicator of void formation. As stated by Lame et al. [8], the aim of such procedure was to follow the void nucleation, rather to assess the precise volume fraction of voids.

The evolution of $Q$ as a function of $\varepsilon_{H}$ was provided in Fig. $S 4 \varepsilon_{H \nu}$ was defined as the value of $\varepsilon_{H}$ where $Q$ starts to increase drastically in the plots in Fig. S4. To provide a comprehensive view about the macroscopic deformation and microscopic void formation, $\varepsilon_{H l}, \varepsilon_{H s}$, and $\varepsilon_{H v}$ of the specimens with various DAs were summarized in Fig. 4. For PPO, $\varepsilon_{H v}$ was 0.04 , slightly smaller than $\varepsilon_{H l}$, suggesting that voids can be formed even at the end of the linear deformation region. For PP15, $\varepsilon_{H v}$ was 0.08 , which was larger than $\varepsilon_{H l}$ but smaller than $\varepsilon_{H s}$. For PP30 and PP45, $\varepsilon_{H v}$ was 0.29 and 0.31 , which were obviously larger than $\varepsilon_{H s}$, implying that voids were initiated in the strain soften region. For PP90, $\varepsilon_{H v}$ was 0.1 and located in between $\varepsilon_{H l}$ and $\varepsilon_{H s}$.

Based on the 2D-SAXS and AFM results, a short summary about the void formation is given as follows: (1) $\varepsilon_{H v}$ is very sensitive to DA. As DA is $0^{\circ}$, voids can be nucleated even slightly earlier before the elastic deformation is finished. As DA is $15^{\circ}$, voids are formed after the elastic deformation but before the strain softening deformation. As DA is $30^{\circ}$ or $45^{\circ}$, voids are induced in the strain softening region. For the specimen with DA of $90^{\circ}$, voids are initiated later than the elastic deformation but earlier than the strain softening deformation; (2) If voids are formed before the strain softening region, the longitude axis would be either perpendicular to the normal of lamellae (PP0, PP15) or parallel to the normal of lamellae (PP90) at the early stage of void formation; If voids are formed in the strain softening region, the longitude direction of voids is perpendicular to the normal of lamellae, although the orientation was 

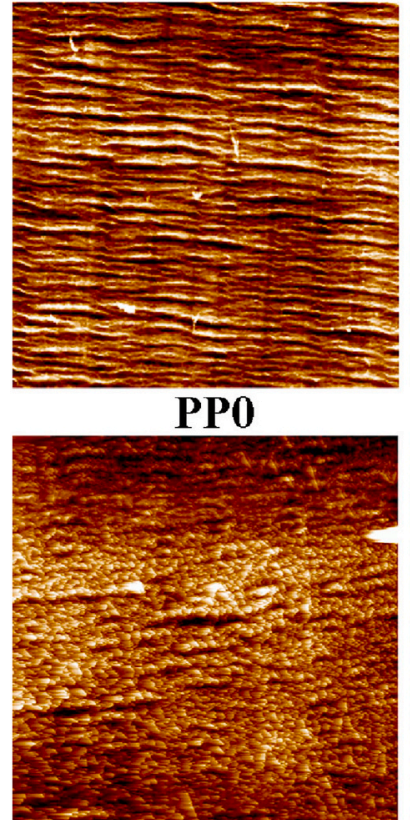

PP45

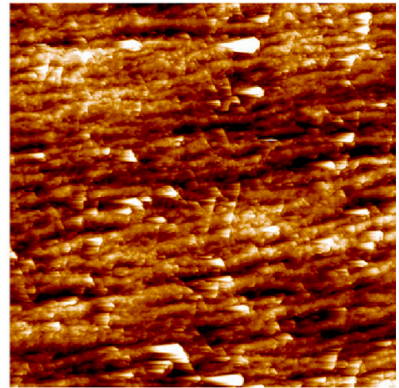

PP15
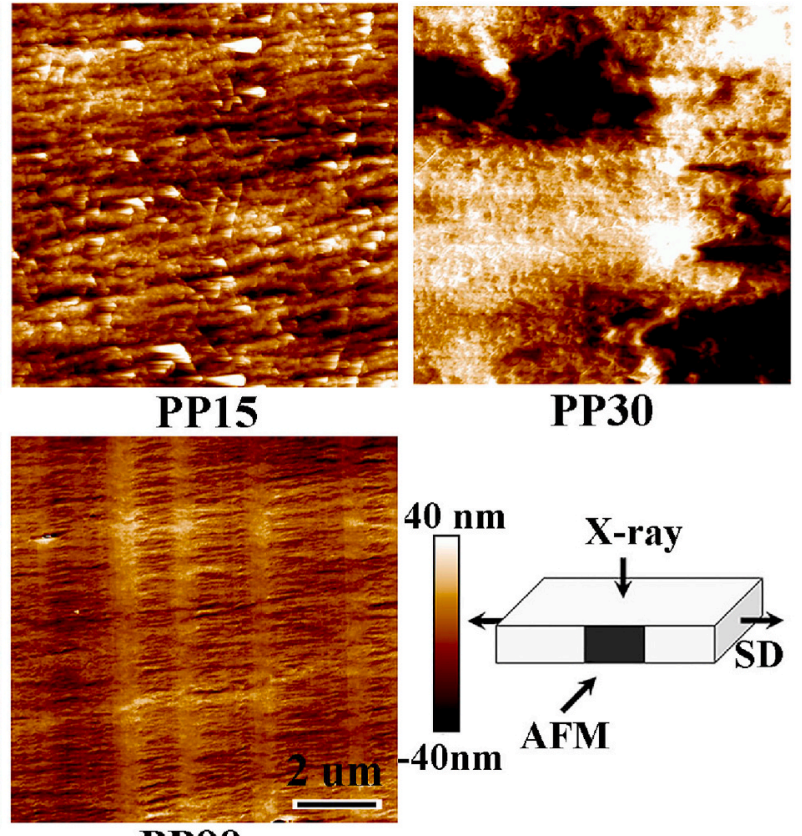

PP30

PP90

Fig. 3. The height diagram of the specimen at the final stage of stretching. SD is horizontal. The image covers a range of $10 \times 10 \mu \mathrm{m}^{2}$.

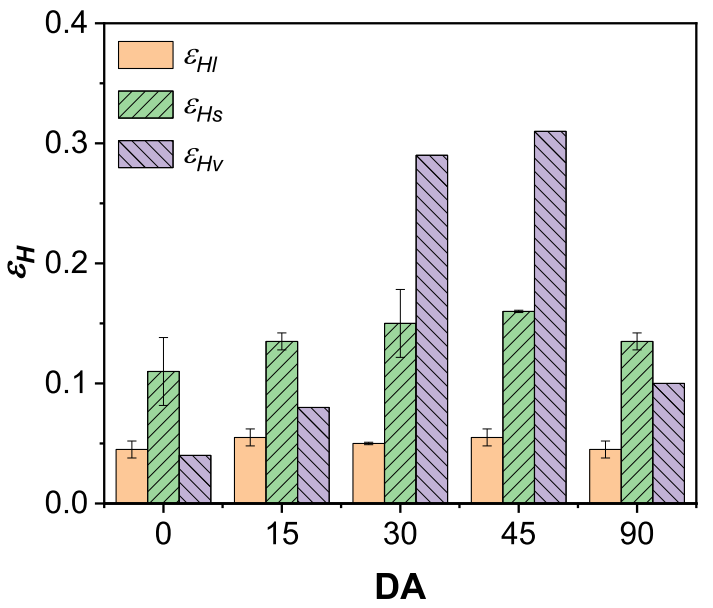

Fig. 4. $\varepsilon_{H l}, \varepsilon_{H s}$, and $\varepsilon_{H v}$ of the samples with different DAs.

very weak (PP30 and PP45). The quite weak orientation of voids when they are induced correlates intimately with their surrounding circumstance of the crystalline phase, where lamellae slip, lamellae rotation, and melting recrystallization happens; (3) In the later stage of stretching, the longitude axis of voids is perfectly aligned in SD, in regardless of its direction when initially formed. To figure out the underlying mechanism, quantitative analyses of the accompanied lamellae and crystal deformation especially at the beginning of voids formation are presented in the following.

\subsection{The slip process of lamellae and chains}

Representative 2D-WAXS patterns during stretching are shown in Fig. 5. One can see that the lattice reflections are concentrated at a specific angle on the pattern, indicating that crystals are oriented in the specimen. The reflections from inner to outer are originated from (110), (040), (130), and (111)/(-131) lattice plane. In addition, typical "crosshatched" structure can be recognized based on the scattering distribution of (110) lattice plane. Taking the un-stretched PP0 as an example, one can see that two-groups of scattering existed on the (110) lattice plane: one is located in the meridian direction, and the other one in the equator direction. Normally, the one in the meridian is ascribed to the "mother" lamellae, and the other one to the "daughter" lamellae. The angle between "mother" and "daughter" lamellae is $c a .80^{\circ}$ [36]. The morphology of the "cross-hatched" structure in single iPP spherulite was also evidenced by scanning electron microscopy in our previous work [37]. Upon stretching, the scattering of lattice plane is re-distributed due to the slip process and melting-recrystallization.

To quantitatively evaluate the slip process, a methodology report by Young et al. [25]. is borrowed here. In their work, three types of slip process were defined, which were fibrillary slip, lamella slip, and chain slip. In the fibrillary slip process, the stacked crystals are organized in fibrils which are capable of shearing relative to each other. Such a shear changes the chain axis and lamella normal towards SD. In the lamella slip, both chain axis and lamella normal rotate together away from SD. In the chain slip process, the chain axis rotated towards SD in most cases the lamella normal remained approximately in its initial direction. Among the three slip processes, chain slip is the only type of deformation where the angle between chain axis and lamella normal changed. By comparing the angle evolution of chain axis and lamella, the dominant slip process can be determined. The directions of chain axis' and the lamellae normal can be obtained from the 2D-WAXS and 2D-SAXS patterns. Since for $\alpha$-iPP, no (001) lattice plane can be found on the 2D-WAXS pattern, then (040) lattice plane is used instead indicating the direction of $b$-axis in the crystal. The angle between $b$-axis and $c$-axis of $\alpha$-iPP is $90^{\circ}$ [38]. The relative change of the azimuthal position of (040) lattice plane and lamellae on 2D-WAXS and 2D-SAXS patterns are labeled as $\Delta \phi_{L}$ and $\Delta \phi_{(040)}$, respectively. The evolution of $\Delta \phi_{L}$ and $\Delta \phi_{(040)}$ with stretching are provided in Fig. 6 . To eliminate the influence of void scattering on the calculation of $\Delta \phi_{L}$, only parts of $\Delta \phi_{L}$ are presented. The positive value in Fig. 6 means that the direction of the lamella normal is moving away from $\mathrm{SD}$, and the direction of $b$-axis is moving towards SD. The star above $x$-axis marks the strain of void formation.

In Fig. 6 one can see that as DA is changed, quite different slip processes can be attributed. In PP0, nearly no change can be seen for either $\Delta \phi_{L}$ or $\Delta \phi_{(040)}$ when voids were formed, suggesting that no slip process appeared when voids were formed. In PP15, $\Delta \phi_{L}$ and $\Delta \phi_{(040)}$ increased 

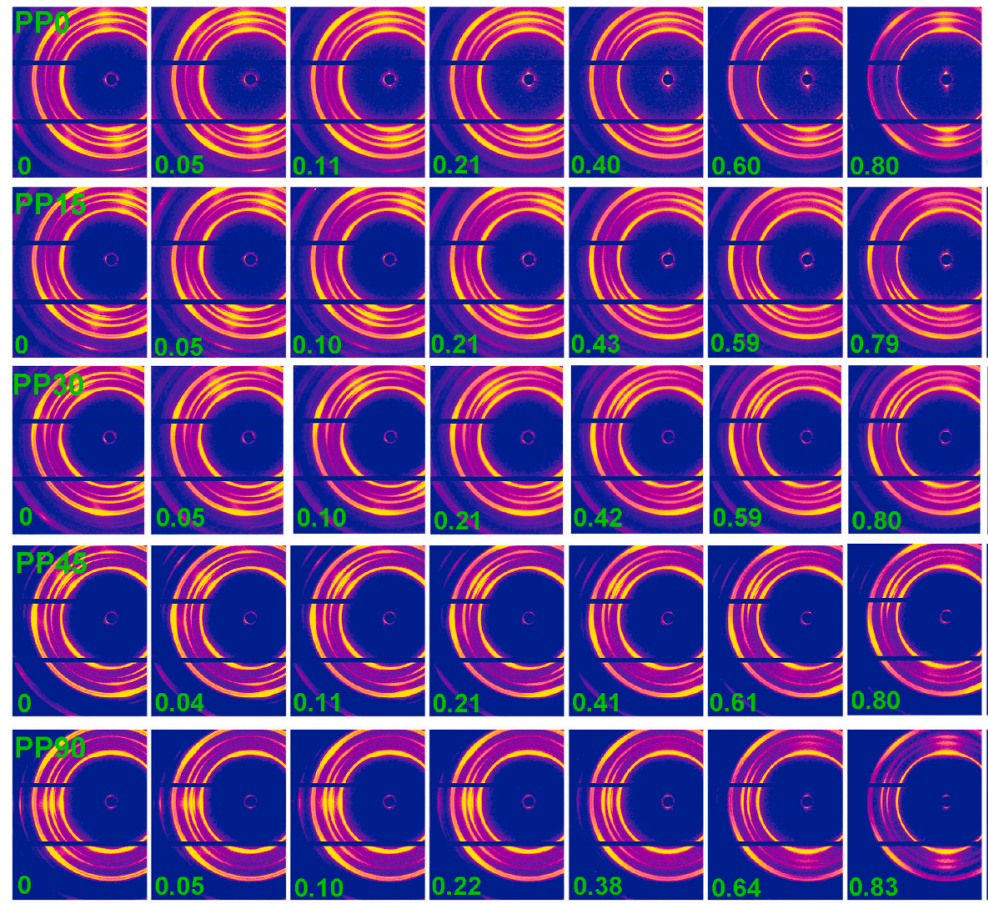
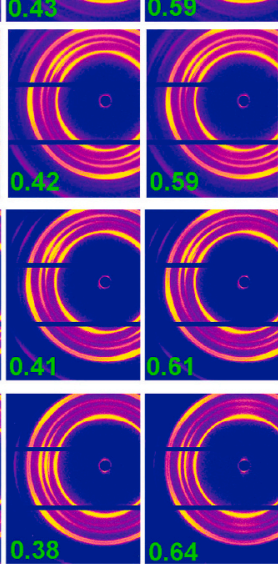
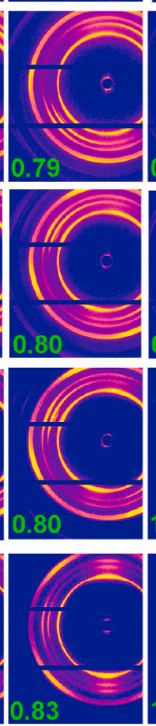
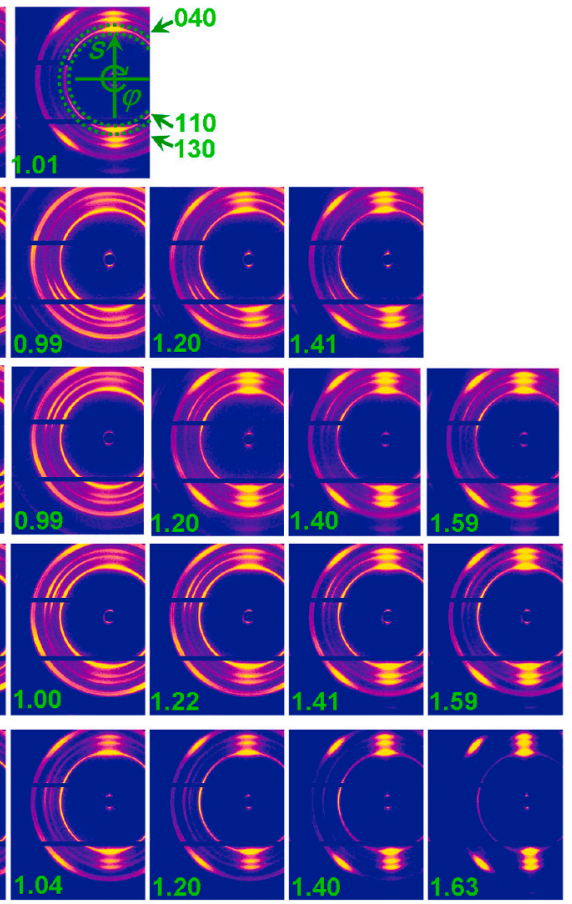

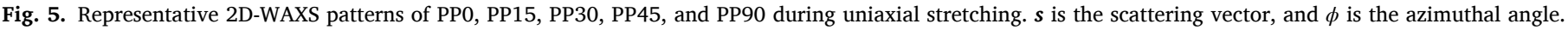

simultaneously with increasing $\varepsilon_{H}$, indicating that the directions of lamella normal and $c$-axis in the crystal were rotating away from SD. At the stretch value $\varepsilon_{H}$ where voids were initiated, $\Delta \phi_{L}$ and $\Delta \phi_{(040)}$ took the value $3^{\circ}$, caused by the slight lamellar slip. In PP30, $\Delta \phi_{L}$ and $\Delta \phi_{(040)}$ were enlarged synchronously when $\varepsilon_{H}$ was smaller than 0.18 . Afterwards, $\Delta \phi_{(040)}$ surpassed $\Delta \phi_{L}$ and the difference between them augments gradually. When voids were activated $\Delta \phi_{L}$ and $\Delta \phi_{(040)}$ are $8.5^{\circ}$ and $10.2^{\circ}$, respectively, meaning that lamellar slip and chain slip coexisted in the system. In PP45, the increasing rate of $\Delta \phi_{L}$ was large than that of $\Delta \phi_{(040)}$ during the whole stretching process, as a result of the synergistic effect of lamellar slip and chain slip, and chain slip dominated the deformation process. At the $\varepsilon_{H}$ where voids were formed, $\Delta \phi_{L}$ and $\Delta \phi_{(040)}$ are $9.0^{\circ}$ and $6.0^{\circ}$. In PP90, similar to PP0 no slip process took place when voids were formed.

\subsection{The microscopic strain of long period}

Due to the existence of hierarchical structures ranging from crystal lattice to lamellae and then further to the packing of lamellae stacks, the macroscopic deformation will not be transmitted in an affine manner to microscopic deformation [39]. In order to get more details about the deformation process in the crystalline phase, the microscopic strain of the long period $\left(\varepsilon_{m l}\right)$ as well as the microscopic strain of the crystal lattice $\left(\varepsilon_{m c}\right)$ are evaluated. The microscopic strain of the long period $\left(\varepsilon_{m l}\right)$ is given as

$L_{p}=1 / s_{\max }$

$\varepsilon_{m L}=\ln \frac{L_{p}}{L_{p}^{0}}$

with $L_{p}$ is the instant long period, $L_{p}^{0}$ is the initial long period before stretching, $s_{\max }$ is the peak position on the Lorentz-corrected intensity distribution curve.

The evolution of $L_{p}$ and $\varepsilon_{m L}$ during stretching are given in Fig. 7a and Fig. 7b, respectively. The green line in Fig. $7 \mathrm{~b}$ is the macroscopic Hencky strain $\varepsilon_{H}$. Before stretching, $L_{p}$ was $19.4 \mathrm{~nm}$. For PP0, as $\varepsilon_{H}$ was increased to $0.04, L_{p}$ grew linearly to $20.2 \mathrm{~nm}$ and $\varepsilon_{m L}$ reached 0.03 correspondingly. The increase of $L_{p}$ is expected since that lamellae stacks with their normal parallel with SD suffers a positive stretching stress. Since that $L_{p}$ comprises the thickness of a lamella and its adjacent amorphous phase, the enlargement of $L_{p}$ can be ascribed to the energy elasticity of the crystal and the entropic elasticity of amorphous phase $[40,41]$. The elastic deformation of the crystal will be studied detailed in the next section. With the further increase of $\varepsilon_{H}, L_{p}$ could not be assessed due to that the voids scattering covers the lamellae signal. For PP15, $L_{p}$ was enlarged linearly to $20.1 \mathrm{~nm}$ and $\varepsilon_{m L}$ grew to 0.04 at a $\varepsilon_{H}$ of 0.05 . The occurrence of lamellae slip seems hardly influence the enlargement of $L_{p}$ as $\varepsilon_{H}$ is smaller than 0.05 . Afterwards, $L_{p}$ continued to increase to $20.4 \mathrm{~nm}$ and $\varepsilon_{m L}$ rose slightly to 0.05 as $\varepsilon_{H}$ reached 0.9 . For PP30, $L_{p}$ grew to $20.4 \mathrm{~nm}$ as $\varepsilon_{h}$ was 0.1 . The increasing rate was smaller than that of PP0 and PP15. As $\varepsilon_{H}$ exceeded 0.1, $L_{p}$ fluctuated with increasing $\varepsilon_{H}$. For PP45, a mild linear increase of $L_{p}$ from $19.2 \mathrm{~nm}$ to $20.0 \mathrm{~nm}$ could be found as $\varepsilon_{H}$ was smaller than 0.3. After that, the increasing of $L_{p}$ was accelerated and $L_{p}$ increased further to $20.9 \mathrm{~nm}$ when $\varepsilon_{H}$ reaches 0.36 . It should be pointed out that in PP30 and PP45, in addition to the stretching of the crystal and polymer chains in amorphous phase, lamellae rotation, lamellae slip and chain slip also contribute the evolution of $L_{p}$. For PP90, different with that of PP0, PP15, PP30, and PP45, $L_{p}$ exhibited a decreasing trend as $\varepsilon_{H}$ was enlarged, indicating the existence of compression stress in the normal direction of lamellae. When $\varepsilon_{H}$ was $0.06, L_{p}$ was reduced to $18.4 \mathrm{~nm}$ and then it stayed constant during further stretching.

\subsection{The microscopic strain of crystal lattice}

The microscopic strain of the crystal lattice $\left(\varepsilon_{m c}\right)$ during deformation is calculated using the following equation

$d_{(\mathrm{hkl})}=1 / s_{(\mathrm{hkl}) \max }$

$\varepsilon_{m c}=\ln \frac{d_{(\mathrm{hkl})}}{d_{(\mathrm{hkl})}^{0}}$

where $d_{(h k l)}^{0}$ and $d_{(h k l)}$ are the initial and instant lattice spacing of the (hkl) lattice plane. $s_{(h k l) \max }$ is the peak position on the intensity distri- 
(a)

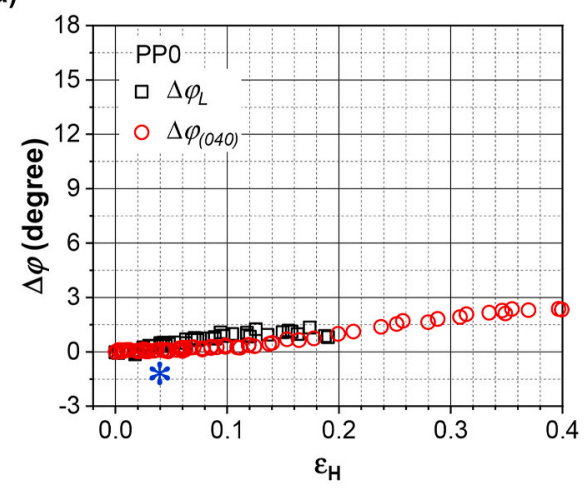

(c)

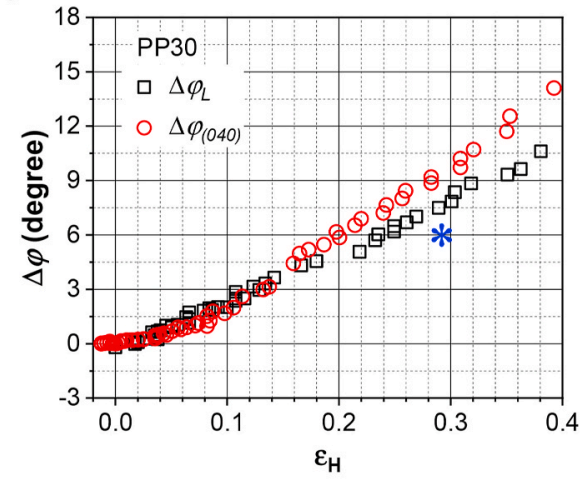

(e)

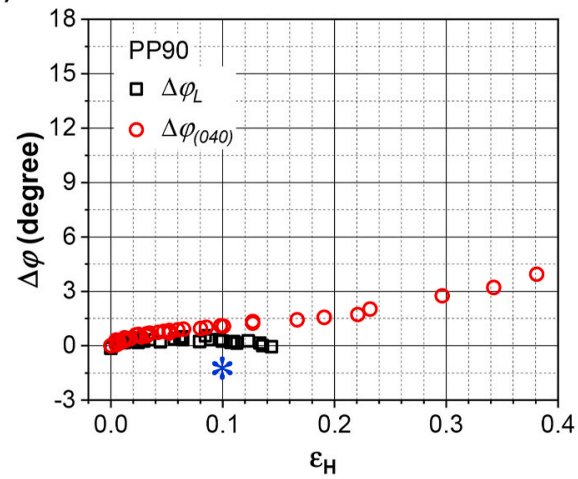

(b)

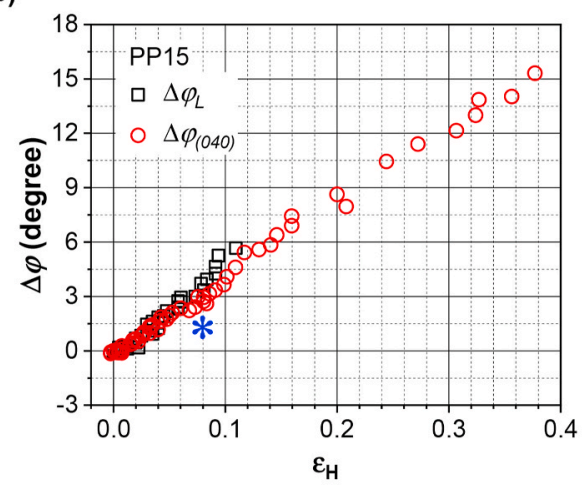

(d)

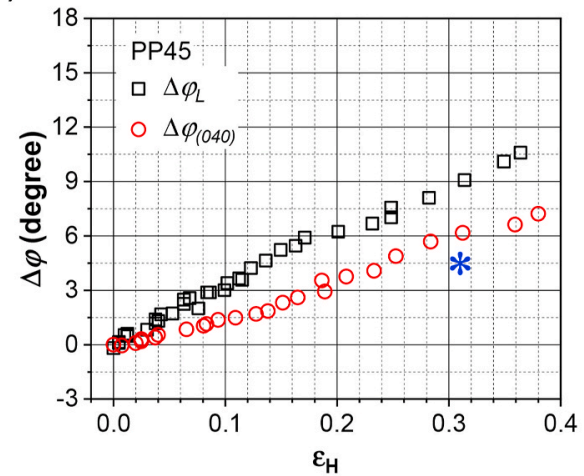

(f)

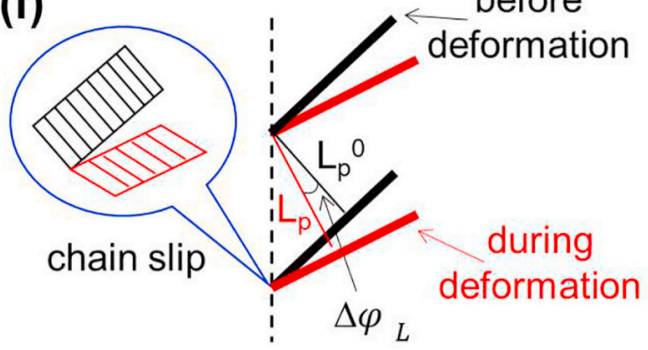

Stretching direction $\Rightarrow$

Fig. 6. The relative change of azimuthal position of lamellae $\left(\Delta \phi_{L}\right)$ and (040) lattice plane $\left(\Delta \phi_{(040)}\right)$ during stretching. The star above $x$-axis marks the strain of void formation.

(a)

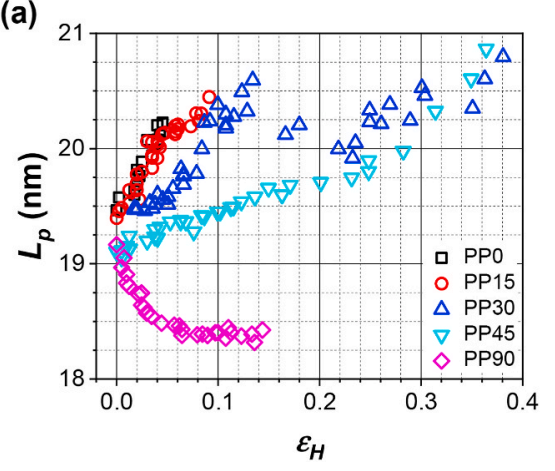

(b)

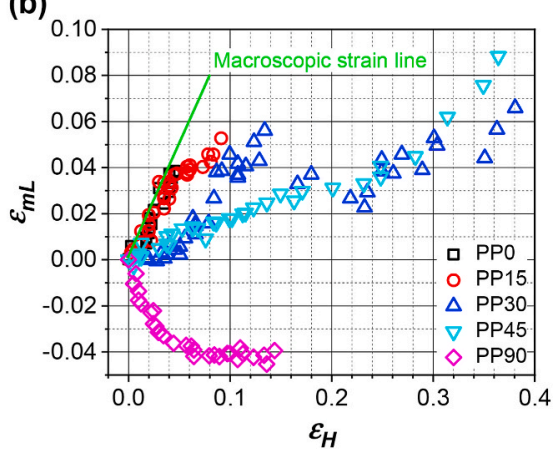

Fig. 7. The evolution of (a) $L_{p}$ and (b) $\varepsilon_{m L}$ with increasing $\varepsilon_{h}$. 
bution curve of (hkl) plane as a function of scattering vector. In this work, the crystal strain of (110) lattice plane and (040) lattice plane is calculated. What's more, as can be seen in Fig. 5, both "mother" lamellae and "daughter" lamellae can be found on (110) lattice plane, so the crystal strains of "mother" lamellae and "daughter" lamellae are calculated respectively. As evidenced in Fig. 6, due to the slip process, the scattering of either (110) lattice plane or (040) lattice plane is shifted in the azimuthal direction. In order to get $s_{(h k l) \max }$ in the scattering vector direction, the following procedure is used (Fig. 8): firstly, an azimuthal scan is performed to locate the peak position in the azimuthal direction $\left(\phi_{\max }\right)$; then 1D-WAXS curve is integrated from a slice of the 2D-WAXS pattern. The azimuthal range of the slice is $\phi_{\max } \pm 2^{\circ}$. The micro strain of (110) lattice plane in "mother" lamellae and "daughter" lamellae are labeled as $\varepsilon_{m c-110 M}$ and $\varepsilon_{m c-110 D}$, respectively. Similarly, the micro strain of (040) lattice plane is labeled as $\varepsilon_{m c-040}$.

Firstly, attention will be focused on the micro strain of the crystal in "mother" lamellae. It can be found in Fig. 9a that with increasing $\varepsilon_{H}, \varepsilon_{m c}$ $110 \mathrm{M}$ of PP0 descended gradually to a minimum value of -0.006 as $\varepsilon_{H}$ was 0.05 . Afterwards $\varepsilon_{m c-110 M}$ enlarged slightly with continuous stretching. The peak value of $\varepsilon_{m c-110 M}$ was much smaller than $\varepsilon_{H}$ or $\varepsilon_{m L}$, which is caused by the fact that the crystalline phase normally owns a much higher modulus than amorphous phase. The modulus of amorphous phase in the rubbery state is expected to be of the order of a few tens of MPa in comparison with the crystal modulus which should be of the order of several tens of GPa [42-44]. In Fig. 9c it can be seen that $\varepsilon_{m c-040}$ of PP0 exhibits a similar trend with that of $\varepsilon_{m c-110 M}$, except the fact that at a same $\varepsilon_{H}$ the absolute value of $\varepsilon_{m c-04}$ was smaller than that of $\varepsilon_{m c-110 M}$. This indicates that in PPO both $a$-axis and $b$-axis were compressed. Taking the Poisson's effect into consideration, the compression of $a$-axis and $b$-axis meant a stretching of $c$-axis along the stretching direction [45]. As DA was increased to $15^{\circ}$, the absolute value of $\varepsilon_{m c-110 M}$ and $\varepsilon_{m c-040}$ became smaller compared with that of PP15 due to the existence of lamellae slip. With the further increase of DA to $30^{\circ}, \varepsilon_{m c-110 M}$ was around 0 and $\varepsilon_{m c-040}$ was slightly higher than 0 , suggesting a compression of $a$-axis and a stretching of $b$-axis. In PP45 and PP90, both $\varepsilon_{m c-110 M}$ and $\varepsilon_{m c-040}$ exhibited a positive value, implying that $a$-axis and $b$-axis suffered a stretching stress, but $c$-axis suffered a compression stress.

Secondly, the deformation of "daughter" lamellae will be discussed. According to the results in Fig. 9b and c, it is inferred that in PPO and PP15 the $a$-axis of the crystal was stretched, but the $b$-axis and the $c$-axis were compressed. The compression of $c$-axis can be attributed to the lateral contraction of the specimen [40]. In PP30 and PP45 the $a$-axis and the $b$-axis were stretched, and the $c$-axis was compressed. Lastly, in PP90 the $b$-axis and the $c$-axis were stretched, but the $a$-axis was compressed.

\subsection{The relative content of "daughter" lamellae during stretching}

The relative content of "daughter" lamellae $\left(X_{D}\right)$ is assessed according to the method proposed by Fujiyama [46]: $X_{D}=\frac{A_{D}}{A_{D}+A_{M}}$, where $A_{D}$ donates the area of "daughter" lamellae and $A_{M}$ donates the area of parent lamellae in Fig. 8b. The azimuthal range used for calculation is 135-315 . The evolution of $X_{D}$ as a function of $\varepsilon_{H}$ is provided in Fig. 10. For PP0, a distinct decrease of $X_{D}$ can be found as $\varepsilon_{H}$ is larger than 0.15 , indicating that "daughter" lamellae are deformed faster than "mother" lamellae. In addition, the critical strain of $X_{D}$ is obviously larger than 0.04 , which marks the critical strain of void formation. When $\varepsilon_{H}$ reaches $0.37, X_{D}$ is further decreased to 0 . The result here suggest that the massive deformation of "daughter" lamellae is later than void formation, which is different with previous report by other researcher [41]. In their research, a drastic decrease of $X_{D}$ from $45 \%$ to $29 \%$ shows up as strain is larger than $10 \%$. The discrepancy may lie in the fact that the stretching temperature and the initial microstructure of the specimen are different. The discrepancy also informs that more work need to be done in future before a consensus is reached. For PP15, PP30, PP45, and PP90 $X_{D}$ shows a very slight linear decrease with increasing $\varepsilon_{H}$, suggesting that "mother" lamellae and "daughter" lamellae are deformed simultaneously upon stretching.

\subsection{The relationship between void formation and crystalline phase deformation}

Combining the results in the above section, a comprehensive discussion about void formation and the deformation of the crystalline phase is given below, and a schematic illustration is provided in Fig. 11:

In PP0 the normal of the "mother" lamellae was arranged in line with $\mathrm{SD}$, and the normal of the "daughter" lamellae was inclined $80^{\circ}$ to SD. During stretching, the long period showed an affine deformation behavior with the macroscopic strain, whereas the crystal strain of "mother" lamellae was much smaller than the macroscopic strain, suggesting that the macroscopic strain was originated mainly by the elastic deformation of the amorphous phase. This could be understood by assuming that the crystalline phase and the amorphous phase were arranged in series, then their deformation could be treated as an iso-stress case [40]. Due to that the modulus of the amorphous phase was much smaller than that of the crystalline phase, so the strain of the amorphous phase should be much larger than that of the crystalline phase. In addition, $\Delta \phi_{L}$ and $\Delta \phi_{(040)}$ remained unchanged suggesting that no slip process happened in the system. The stress applied on the specimen can be effectively transferred to the amorphous phase. As $\varepsilon_{H}$ reached 0.04 , the negative stress inside the amorphous phase was higher than the critical stress needed for void formation. Consequently, voids were triggered in the amorphous phase. Additionally, once void was induced, its tip will propagate quickly in the direction perpendicular to the (a)

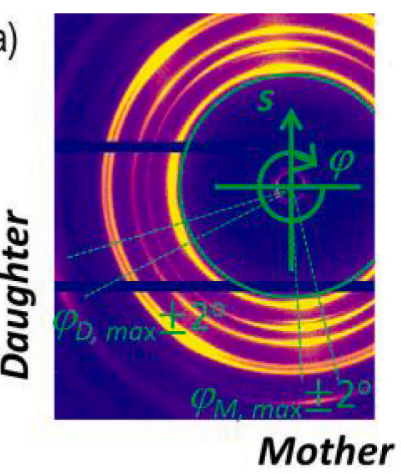

(b)

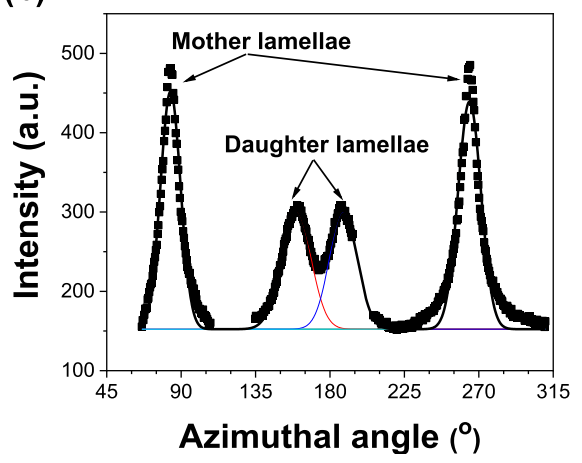

(c)

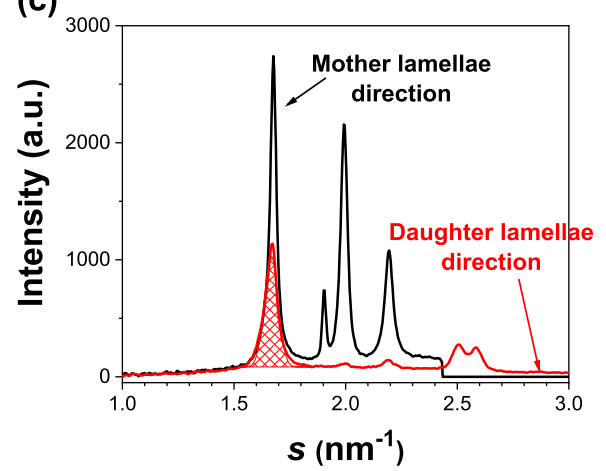

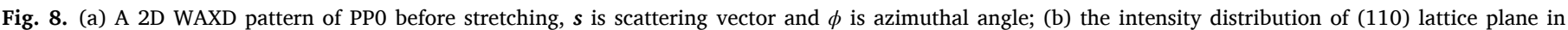

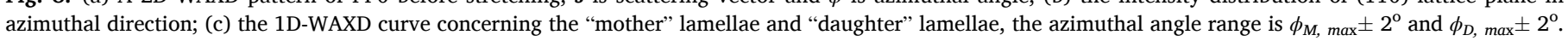


(a)

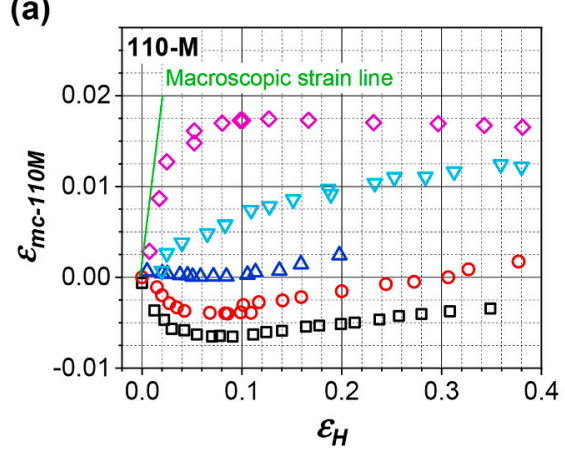

(b)

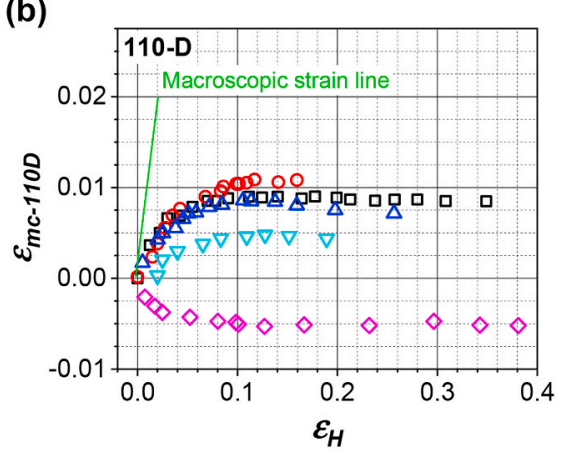

(c)

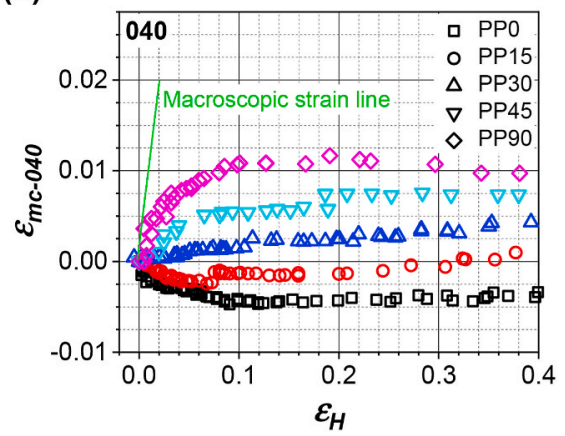

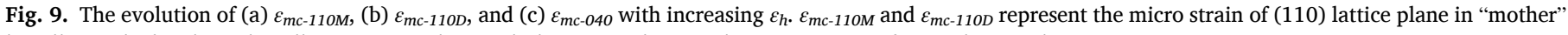
lamellae and "daughter" lamellae, respectively. Similarly, $\varepsilon_{m c-040}$ donates the micro strain of (040) lattice plane.

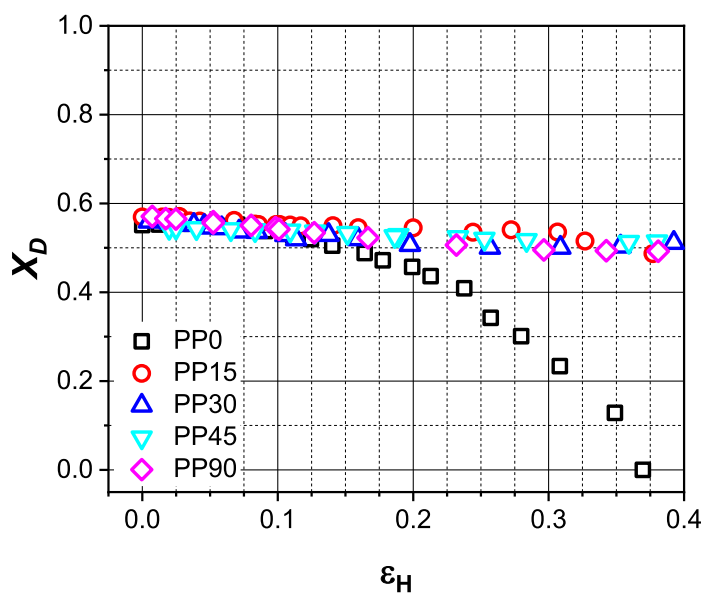

Fig. 10. The evolution of the relative content of "daughter" lamellae $\left(X_{D}\right)$.

stretching stress. So, the longitude direction of voids appeared perpendicular to the stretching direction, as shown in Fig. 2. The propagation of void tip was of great possibility caused by the disentanglement of polymer chains ahead of the void tip [24,47-49].

In PP15, the normal of "mother" lamellae was inclined $15^{\circ}$ to SD. Once the stretching was performed, $\Delta \phi_{L}$ and $\Delta \phi_{(040)}$ were increased synchronously, indicating that lamellae rotation and lamellae slip were triggered. The occurrence of lamellae rotation and slip consumed part of the work applied on the specimen. The microscopic strain of the long period was nearly the same with that of PP0, but the microscopic strain of the crystal was smaller. Regarding the system as an iso-stress case, a smaller microscopic strain of the crystal means that the stress in the amorphous phase became smaller compared with that of PPO. Therefore, voids were induced at a larger critical strain in PP15 [25].

In PP30 and PP45, both $\Delta \phi_{L}$ and $\Delta \phi_{(040)}$ were increased with the enlargement of $\varepsilon_{H}$. And different with that in PP15, a deviation appeared between the increment of $\Delta \phi_{L}$ and $\Delta \phi_{(040)}$, suggesting that in addition to lamellae slip, chain slip also took place. Therefore, more energy was consumed before voids were induced. In addition, based on the results in Fig. 9, when voids were induced, in the normal direction the "mother" lamellae suffered a quite weak stretching stress (PP30), or even a compression stress (PP90). Hence the orientation of voids was quite weak.

In PP90, the normal of "mother" lamellae was perpendicular to SD. During stretching, neither lamellae slip nor chain slip occurred when voids were induced. So, the critical strain of void formation was much smaller than that of PP30 and PP45. Besides, in their normal direction, "mother" lamellae suffered a compression stress (evidenced by the crystal strain in Fig. 9), voids were impossible to be induced in the amorphous phase on the normal side of lamellae. Therefore, it is speculated that voids were induced in the amorphous phase on the lateral side of "mother" lamellae. In addition, according to the result in Fig. 3, it can be found that compared with PP0, the void number was much larger and the void size was much smaller in PP90.

\section{Conclusion}

By changing the DA, the relationship between void formation and plastic deformation of crystalline phase during uniaxial stretching in oriented iPP was studied in this work. Results show that the occurrence of lamellae slip or chain slip presents a retardation effect on void formation. As the DA is $0^{\circ}$, voids are initiated at a $\varepsilon_{H}$ of 0.04 and the longitude direction of voids is perpendicular to the normal of lamellae. In addition, no slip process appears when voids were induced. As DA is $15^{\circ}$, lamellae slip took place once the stretching was started and voids were formed at $\varepsilon_{H}$ of 0.09 . When DA is further enlarged to $30^{\circ}$ and $45^{\circ}$, both lamellae slip and chain slip is observed as voids were induced. The corresponding $\varepsilon_{H}$ of voids formation were 0.29 and 0.31 , respectively. Interestingly, it is worth noting that as DA was increased from $0^{\circ}$ to $45^{\circ}$, although the orientation degree of voids was decreased, the longitude 

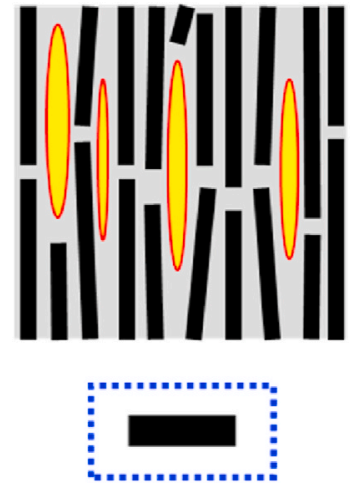

lamella
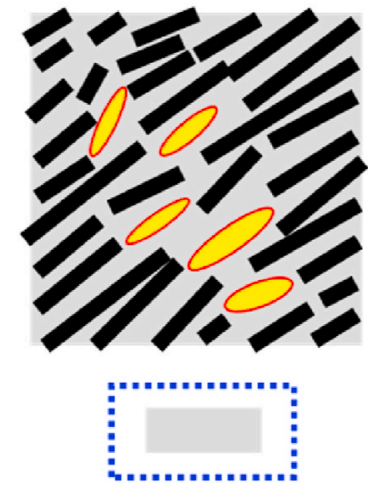

amorphous phase
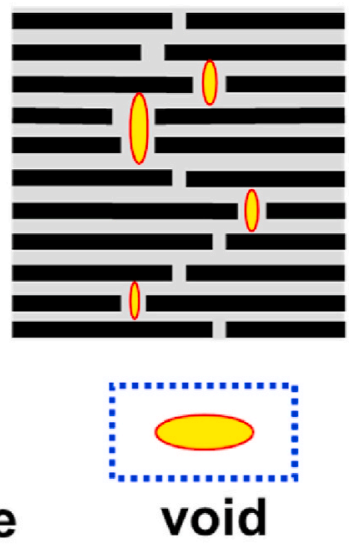

\section{Stretching direction}

Fig. 11. A schematic illustration about void formation in oriented iPP.

direction of voids stayed perpendicular to the normal of lamellae. When DA was increased to $90^{\circ}$, voids were formed at $\varepsilon_{H}$ of 0.1 and the longitude direction of voids coincided with the lamellae normal. Additionally, neither lamellae slip nor chain slip existed once voids appeared. Based on investigation of the initial direction of voids, it is proposed that voids were induced either in the amorphous phase on the normal side of lamellae $\left(0^{\circ} \leq \mathrm{DA} \leq 45^{\circ}\right)$ or in the amorphous phase on the lateral side of lamellae $\left(\mathrm{DA}=90^{\circ}\right)$.

\section{Declaration of competing interest}

The authors declare that they have no known competing financial interests or personal relationships that could have appeared to influence the work reported in this paper.

\section{Acknowledgement}

The authors are grateful to DESY for beamtime within the project II20150042. Baobao Chang thanks the Grant by China Scholarship Council. Baobao Chang gratefully acknowledges the financial support for this work by the National Natural Science Foundation of China (grant no. 52003249), the China Postdoctoral Science Foundation (grant no. 2019M652571), the Natural Science Foundation of Henan (grant no. 202300410426), the Open Funding Project of National Key Laboratory of Human Factors Engineering (grant no. SYFD061910K).

\section{Appendix A. Supplementary data}

Supplementary data to this article can be found online at https://doi. org/10.1016/j.polymer.2020.123342.

\section{References}

[1] C.W. Barney, C.E. Dougan, K.R. McLeod, A. Kazemi-Moridani, Y. Zheng, Z. Ye, S. Tiwari, I. Sacligil, R.A. Riggleman, S. Cai, J.-H. Lee, S.R. Peyton, G.N. Tew, A J. Crosby, Proc. Natl. Acad. Sci. Unit. States Am. 117 (17) (2020) 9157-9165.

[2] P.I. Vincent, Polymer 1 (1960) 7-19, 0.

[3] T. Wu, M. Xiang, Y. Cao, J. Kang, F. Yang, RSC Adv. 4 (69) (2014) 36689-36701.

[4] A. Pawlak, A. Galeski, A. Rozanski, Prog. Polym. Sci. 39 (5) (2014) 921-958.

[5] A. Pawlak, A. Galeski, Macromolecules 38 (23) (2005) 9688-9697.

[6] Y. Lu, Y. Men, Macromol. Mater. Eng. 303 (11) (2018) 1800201-1800231, 1800203.

[7] Y. Lu, Y. Wang, R. Chen, J. Zhao, Z. Jiang, Y. Men, Macromolecules 48 (16) (2015) 5799-5806.

[8] S. Humbert, O. Lame, J.M. Chenal, C. Rochas, G. Vigier, Macromolecules 43 (17) (2010) 7212-7221.
[9] Y. Lin, X. Li, L. Meng, X. Chen, F. Lv, Q. Zhang, R. Zhang, L. Li, Macromolecules 51 (7) (2018) 2690-2705.

[10] C. Lei, R. Xu, Z. Tian, H. Huang, J. Xie, X. Zhu, Macromolecules 51 (9) (2018) 3433-3442.

[11] A. Saffar, P.J. Carreau, A. Ajji, M.R. Kamal, Ind. Eng. Chem. Res. 53 (36) (2014) 14014-14021.

[12] B. Chang, K. Schneider, F. Xiang, R. Vogel, S. Roth, G. Heinrich, Macromolecules 51 (16) (2018) 6276-6290.

[13] D. Lyu, R. Chen, Y. Lu, Y. Men, Ind. Eng. Chem. Res. 57 (27) (2018) 8927-8937.

[14] B. Chang, K. Schneider, R. Vogel, G. Heinrich, Polymer 138 (28) (2018) 329-342.

[15] Y. Lu, Y.-F. Men, Chin. J. Polym. Sci. 36 (10) (2018) 1195-1199.

[16] F. Auriemma, C. De Rosa, R. Di Girolamo, A. Malafronte, M. Scoti, G.R. Mitchell, S. Esposito, Macromolecules 50 (7) (2017) 2856-2870.

[17] Y. Wang, Z. Jiang, L. Fu, Y. Lu, Y. Men, PLOS ONE 9 (5) (2014), e97234, 97231 97214.

[18] A. Krajenta, A. Rozanski, J. Polym. Sci. B Polym. Phys. 54 (18) (2016) 1853-1868.

[19] Y. Lin, X. Li, X. Chen, M. An, Q. Zhang, D. Wang, W. Chen, P. Yin, L. Meng, L. Li, Polymer 178 (2019) 121579.

[20] J. Zhou, Y. Zheng, G. Shan, Y. Bao, W.-J. Wang, P. Pan, Polymer 188 (2020) 122121.

[21] Z.W. Cai, Y. Zhang, J.Q. Li, F.F. Xue, Y.R. Shang, X.H. He, J.C. Feng, Z.H. Wu, S. C. Jiang, Polymer 53 (7) (2012) 1593-1601.

[22] A. Rozanski, A. Galeski, M. Debowska, Macromolecules 44 (1) (2011) 20-28.

[23] A. Rozanski, A. Galeski, Macromolecules 44 (18) (2011) 7273-7287.

[24] A. Pawlak, J. Krajenta, A. Galeski, Polymer 151 (2018) 15-26.

[25] R.J. Young, P.B. Bowden, J.M. Ritchie, J.G. Rider, J. Mater. Sci. 8 (1) (1973) 23-36.

[26] S.G. Burnay, G.W. Groves, J. Mater. Sci. 13 (3) (1978) 639-646.

[27] A. Peterlin, J. Mater. Sci. 6 (6) (1971) 490-508.

[28] Z. Wang, Y. Liu, C. Liu, J. Yang, L. Li, Polymer 160 (2019) 170-180.

[29] X. Yang, B. Dong, Y. Shang, Y. Ji, F. Su, C. Shao, Y. Wang, C. Liu, C. Shen, Polymer 194 (2020) 122385.

[30] R. Hiss, S. Hobeika, C. Lynn, G. Strobl, Macromolecules 32 (13) (1999) 4390-4403.

[31] S. Hobeika, Y. Men, G. Strobl, Macromolecules 33 (5) (2000) 1827-1833.

[32] Y. Men, J. Rieger, G. Strobl, Phys. Rev. Lett. 91 (9) (2003), 095502.

[33] A.F. Thünemann, W. Ruland, Macromolecules 33 (5) (2000) 1848-1852.

[34] Z. Jiang, T. Liao, R. Chen, Y. Men, Polymer (2019) 121984.

[35] Y. Lu, Y. Wang, R. Chen, Y. Men, J. Polym. Sci. B Polym. Phys. 55 (12) (2017) 957-963.

[36] D.R. Norton, A. Keller, Polymer 26 (5) (1985) 704-716.

[37] B. Chang, K. Schneider, N. Patil, S. Roth, G. Heinrich, Polymer 142 (2018) 387-393.

[38] B. Lotz, Polymer 39 (19) (1998) 4561-4567.

[39] C. Millot, R. Séguéla, O. Lame, L.A. Fillot, C. Rochas, P. Sotta, Macromolecules 50 (4) (2017) 1541-1553.

[40] H. Zhou, G.L. Wilkes, J. Mater. Sci. 33 (2) (1998) 287-303.

[41] X. Chen, R. Xu, J. Xie, Y. Lin, C. Lei, L. Li, Polymer 94 (2016) 31-42.

[42] Y. Shi, C. Zheng, M. Ren, Y. Tang, L.-Z. Liu, B. He, Polymer 123 (2017) 137-143.

[43] K. Tashiro, M. Kobayashi, H. Tadokoro, Polym. J. 24 (9) (1992) 899-916.

[44] M.R. Viotti, G.H. Kaufmann, G.E. Galizzi, Optic Laser. Eng. 44 (6) (2006) 495-508.

[45] B. Xiong, O. Lame, R. Seguela, Y. Men, Polymer 140 (2018) 215-224.

[46] M. Fujiyama, T. Wakino, Y. Kawasaki, J. Appl. Polym. Sci. 35 (1) (1988) 29-49.

[47] C.B. Bucknall, Polymer 48 (4) (2007) 1030-1041.

[48] A.S. Argon, Polymer 52 (10) (2011) 2319-2327.

[49] D.S.A. De Focatiis, C.P. Buckley, Polymer 52 (18) (2011) 4045-4053. 